International Journal of Advances in Pharmacy and Biotechnology

Vol.4, Issue-3, 2018, 1-5

Research Article

Open Access.

I J A P B

ISSN: 2454-8375

\title{
EVALUATION OF BIOCHEMICAL MARKERS FOR BONE CHANGES IN LEPROSY PATIENTS
}

\author{
Yaswanth Kumar K¹, Alekya G¹, Manasa Sowmya K², Venkateshwarlu Eggadi1, \\ Sharavanabhava B.S $\mathbf{1}^{*}$
}

${ }^{1}$ Department of Clinical Pharmacy \& Pharm.D., Vaagdevi College of Pharmacy, Warangal, Telangana, Kakatiya University- 506001, India.

2Manisha Neuropsychiatry Clinic, Kakaji Colony, Hanamkonda, Warangal-506007, Telangana, India. Institute of Pharmaceutical Sciences, Visakhapatnam-531173, A.P., India.

*Corresponding author e-mail: sharvanabhava6@gmail.com

Received: 10 August 2018

Revised: 20 August 2018

Accepted: 25 August 2018

\begin{abstract}
:
Leprosy has prominent effect on hands and feet. Bone deformities are the most occurred complication in leprosy and can result to deformity and disability. Bone resorption leads to the increased levels of ALP, U. $\mathrm{CA} / \mathrm{CR}$ level ratio. Decreased hemoglobin level is most observed in these patients. The leprosy patients were compared with healthy. U. CA/CR levels is used for studying bone resorption which may be associated with new bone formation it can be evaluated by estimating ALP levels. Blood changes is also studied by estimating Haemoglobin in Hansen's patients. The mean value U.CA/CR ratio, ALP is also increased in cases when compared to controls which indicate bone resorption coupled with compensatory new bone formation. The $\mathrm{Hb} \%$ was also decreased in leprosy patients. The present study suggests an increased bone resorption in leprosy patients and is attributed to compensatory new bone formation coupled with bone resorption. In conclusion our data suggests that therapy aimed at reducing bone loss may benefit these patients with high bone turnover and decrease the deformities and disease burden due to leprosy.
\end{abstract}

Key words: Leprosy, Bone resorption, alkaline phosphatase (ALP), Urinary Calcium/Creatinine level ratio.

\section{INTRODUCTION:}

Leprosy (Hansen's disease) is a steadily intensifying, contagious, chronic granulomatous disease precipitated by Mycobacterium leprae. Most frequently affected are skin and superficial peripheral nerves. Bone involvement is one of the principal forecasting factors occurs in 15\% to $29 \%$ of patients [1]. Myocardium leprae was the first microorganism associated with a specific disease. It is a non-motile, non-spore forming, microaerophilic, acid-fast staining bacterium and generally forms slightly curved (or) straight rods [2].
M. leprae was discovered by Hansen in 1873, the first bacterium to be identified as causing disease in humans [3] .It is carried via droplets from the nose and mouth, through close contact with a affected person who has not received treatment. Incubation period of M. leprae range from 3 to 7 years. However leprosy has been eliminated from many areas all over worldwide, its prevalence is still elevated in endemic areas [4].There is a practical classification which was proposed by Ridley and Jopling in 1966 based on the principles described by skinsnes (1964) named as 1. Polar tuberculoid (TT) 2. Borderline tuberculoid (BT) 3. Mid borderline (BB) 4. Borderline lepromatous (BL) 5. Polar 
lepromatous (LL) Bone deformities are the commonly occurred complication in leprosy. The frequency of bone changes which has been recorded in different studies has varied from $15 \%$ to $95 \%$ [5]. M. leprae prominently affects hands and feet. It also affects bones of face and in more advanced cases, bones of cranium or axial skeleton. Small bone cysts, enlargement of nutrient canals are seen due to leprae bacilli invasion to nerves which are supplying arterioles of the bone. Lechat proposed a classification for bone changes in leprosy and it can be divided into two major groups: 1. Specific leprous osteitis 2. NonSpecific bone changes [6]. Specific type of bone changes in leprosy results by direct invasion of bones by M. leprae. Specific type of bone lesions are very rare, and effects small bones of hands, feet and nose which is seen in lepromatous forms. Non-Specific type of bone changes are secondary to the obstruction of nerve supply leading to vascular changes, trauma and secondary infection. Non-Specific type of bone lesions are very common and are classified as 4 types 1. Distal absorption of the digits 2 . osteoarthritis $3 . \quad$ osteomyelitis 4 . osteoporosis. Bone resorption is a major condition in leprosy which in early stages of disease [7]. Bone resorption may result in permanent deformities which act as major cause for morbidity. Almost 1/4th proportion of leprosy patients who are not treated at early stages of the disease develop deformities of hands and feet. Most important factor in bone destruction in leprosy is ulceration which is frequently followed by secondary infection and pyogenic osteomyelitis. Ulceration is usually associated with infection of soft tissues, in plantar surfaces of feet which is caused by circulatory disturbances effected on long standing pressure in distal parts of the extremities due to neural involvement by leprosy as well as to acute injury. Radiologic studies are useful in finding bone resorption in leprosy [8]. Bone resorption involving face alveolar bones and limbs in leprosy patients can be determined by radiological studies [9]. Facial deformities include nasal deformities, septal perforation along with eye deformities and facial nerve palsy has been analyzed in clinical study [10]. Archaeological studies have found bone resorption in skeleton of leprosy [11]. Patients with few biochemical markers have been made to determine bone resorption in leprosy with conflicting results [12]. Bone is a dynamic tissue characterized by continuous genesis and destruction. Bone formation occurs in growing bone. Bone resorption occurs in aging bone which results in osteoporosis and osteopenia [13]. Bone turnover can be determined by many biochemical markers [14].

\section{METHOD}

In this study subjects include ages of 10-80 years with both genders (Male \& Female) of population suffering from Hansen's disease. The present study is a Prospective Observational and Case-Control study conducting at Department of Dermatology, Venerology and Leprosy, MGM Hospital, Warangal, Telangana, India.Fourty leprosy diagnosed patients were selected. Boneresorption is studied by measuring fasting urinary calcium, creatinine level. Bone resorption may be associated with new bone formation. So, serum alkaline phosphatase (ALP) was estimated as a marker of bone formation. Blood changes are also studied by estimating haemoglobin percentage in these patients. Evaluation of bone changes by X-ray in patients diagnosed with leprosy. Follow-up for these patients was taken to evaluate the new bone formation by prescribing calcium supplements. 
Inclusion Criteria: Both males and females of age 10-80 years. Patients diagnosed with any type of leprosy. Healthy individuals are also taken as standard group.

Exclusion Criteria: Pregnant and lactating women. Patients already suffering from bone deformities. Patients with a personal or family history of osteometabolic disease, clinical and laboratory evidences of hepatic and renal disease.

\section{RESULTS}

A total number of 60 individuals have been enrolled in the study out of which 40 patients were diagnosed with leprosy and 20 were healthy individuals. All the 60 individuals who are included in the study were above 10 years and below 80 years of age (Table 1).

Table 1: Gender wise distribution of the patients suffering from different types of leprosy

\begin{tabular}{lcr}
\hline \multicolumn{1}{c}{ Gender } & $\begin{array}{c}\text { Group-I } \\
\text { No. of Patients (\%) }\end{array}$ & $\begin{array}{c}\text { Group-II } \\
\text { Control (\%) }\end{array}$ \\
\hline Male & $13(32.5)$ & $8(40)$ \\
Female & $27(67.5)$ & $12(60)$ \\
\hline
\end{tabular}

Comparison of bone changes in leprosy patients using following parameters among the patients (group I) and control (group II): The mean values of Urinary $\mathrm{Ca} / \mathrm{Cr}$ were significantly increased in cases when compared to controls. The increased calcium excretion in urine of leprosy patients as observed in present study is due to underlying increase in bone resorption.

The amount of creatinine excreted in urine is usually constant and is little influenced by the diet. The mean values of Serum Alkaline Phosphatase were significantly increased in cases when compared to controls. The raised Serum Alkaline Phosphatase activity in Cases is attributed to compensatory new bone formation coupled with bone resorption, urinary calcium, creatinine ratio, serum alkaline phosphatase. The mean values of Urinary $\mathrm{Ca} / \mathrm{Cr}$ were significantly increased in cases when compared to controls. The increased calcium excretion in urine of leprosy patients as observed in present study is due to underlying increase in bone resorption. The amount of creatinine excreted in urine is usually constant and is little influenced by the diet. The mean values of serum alkaline phosphatase were significantly increased in cases when compared to controls. The raised serum alkaline phosphatase activity in cases is attributed to compensatory new bone formation coupled with bone resorption. The amount of creatinine excreted in urine is usually constant and is little influenced by the diet. The mean values of serum alkaline phosphatase were significantly increased in cases when compared to controls. The raised Serum Alkaline Phosphatase activity in Cases is attributed to compensatory new bone formation coupled with bone resorption (Table 2).

Comparison of hemoglobin changes in leprosy patients (group I) and control (group II):

The mean values of hemoglobin were significantly decreased in leprosy patients when compared to controls (Table 2).

\begin{tabular}{|c|c|c|c|c|}
\hline Parameters & Group-I & Group-II & 't' Value & 'p' Value \\
\hline $\begin{array}{l}\text { Urinary Calcium/ } \\
\text { Creatinine }\end{array}$ & $0.2043 \pm 0.007$ & $0.149 \pm 0.0354$ & 2.037 & $0.0462^{*}$ \\
\hline $\begin{array}{l}\text { Serum Alkaline } \\
\text { Phosphatase }\end{array}$ & $97.49 \pm 4.128$ & $82.9 \pm 5.304$ & 2.101 & $0.0400^{*}$ \\
\hline Hemoglobin & $9.67 \pm 0.324$ & $11.45 \pm 0.276$ & 3.558 & $0.0008^{* * *}$ \\
\hline
\end{tabular}




\section{DISCUSSION}

In the present study we aimed to evaluate bone changes in leprosy patients by using some biochemical markers and X-ray reports. Leprosy has a multisystem involvement that leads to wide range of biochemical changes in the body. Bone resorption is an early event in leprosy. The amount of bone resorption may vary depending on severity of the disease. In the present study prevalence of leprosy is more in the age of 31-40 years and female population are much more affected by bone resorption. In this bone resorption was studied by measuring fasting urinary calcium/ creatinine level. So, Serum Alkaline Phosphatase activity was estimated as a marker of bone formation. The study conducted by comparison between patient and controls were done by measuring serum alkaline phosphatase and Urinary Calcium/ Creatinine levels but in their study only increased mean difference for U.CA/CR but there was no statistical significant difference between two groups [3]. From the present study, it was observed that there was a statistically significant increase in mean value of both Urinary Calcium/ Creatinine ratio (0.2043 Vs 0.1497) and Serum Alkaline phosphatase activity (97.49 Vs 82.9) in patients compared to controls.

The increased calcium excretion in urine of leprosy patients as observed in present study may be due to underlying increase in bone resorption. The amount of creatinine excreted in urine is usually constant and is little influenced by the diet. In the present study, the increase in Urinary Calcium/ Creatinine in leprosy cases was associated with duration, bacterial load and type of disease. There is a statistically significant increase in Urinary Calcium/ Creatinine and serum alkaline phosphatase activity in patients which indicates bone resorption and bone formation [6].
Blood changes are also hall marks of leprosy in which hemoglobin percentage will be decreased. The amount of decreased hemoglobin depends on severity of disease. In the present study, it was observed that there was a statistically significant decrease in mean value of $\mathrm{Hb} \%$ (9.67 Vs 11.45) in patients as compared to Controls. The raised Serum Alkaline Phosphatase activity in Cases is attributed to compensatory new bone formation coupled with bone resorption. The present study suggests an increase in bone turnover in leprosy. The etiology of increased bone resorption is multi factorial. It was proposed that bone loss in patients with leprosy is an acceleration of a normal cellular process and could be due to local release of products from M. leprae or host cells. Cytokines released from immune cells of host in response to infection may play a role in bone resorption [8].

As Multi Drug Therapy in leprosy has limited impact on bone loss, specific therapeutic treatment aimed at bone resorption have been studied by Kanji et al., and they have found the need for Anti-Resorptive Drugs along with Multi Drug Therapy in leprosy. In leprosy administration of Calcium Supplements may be of benefit as an additional therapeutic measure to retard the progress of bone changes characteristics of the disease. This is allied to the study conducted by [3]. In the present study there was a significant decrease in P-Value for Urinary Calcium/Creatinine, Serum Alkaline Phosphatase and Haemoglobin.

\section{ACKNOWLEDGEMENT}

Authors are thankful to the Principal and Secretary, Vaagdevi college of Pharmacy, for providing necessary facilities and actions towards the fruitful completion of this research work. 


\section{CONFLICT OF INTEREST}

The author(s) confirm that this article content has no conflict of interest.

\section{REFERENCES}

[1] Ankad BS, Halawar RS. Bone involvement in leprosy: Early changes. Radiology of Infectious Diseases. 2015 Mar 1;1(2):88-9.

[2] Silva MC, Verano J. Bone changes and immune response in leprosy.

[3] Swathi M, Rao JR, Silvia CR. Evaluation of bone resorption markers in leprosy. Int J ClinDiag Res. 2014;2:1-7.

[4] EichelmannKSE, González González, JC, Salas-Alanis J, Ocampo-Candiani .Leprosy. An Update : Definition, Pathogenesis, Classification, Diagnosis, and Treatment. ActasDermosifiliogr 2013; 104(7): 554-563.

[5] Chandanwale AS, Shivkumar, Pande G. Leprosy and orthopaedic complications - Current status in India. International Journal of Research and Reviews in Pharmacy and Applied science 2012; 2(6): 11201127.

[6] Skinsnes OK, Sakurai I, Aquino TI. Pathogenesis of extremity deformity in leprosy. Int J Lebr. 1972;40:375-88.

[7] Marks SC Jr. The cellular basis for extremity bone loss in leprosy. International Journal of Leprosy and Other Mycobacterial Diseases 1979; 47(1): 26-32.

[8] Changes in Leprosy. A study of 50 cases Indian Journal of Leprosy 1985;
57(3): 632-639 Chhabriya BD, Sharma NC, Bansal NK, Agrawal GR. Bone

[9] Subramaniam K, Nah SH, Marks SC. A longitudinal study of alveolar bone loss around maxillary central incisors in patients with leprosy in Malaysia. Leprosy Review 1994; 65(2):137-142

[10] Ji Heui Kim, Ok Joo Lee, Jae Jun Lee, Chan Hum Park. Analysis of Facial Deformities in Korean Leprosy. Clinical and Experimental Otorhinolaryngology 2013; 6(2): 7881.

[11] G. Michael Taylor, Stephanie Widdison, Ivor N. Brown, Douglas Young. A Mediaeval Case of Lepromatous Leprosy from 13-14th Century Orkney, Scotland. Journal of Archaeological Science 2000; 27: 1133-1138.

[12] Ishikawa A, Ishikawa S, Hirakawa M. Osteoporosis, bone turnover and hypogonadism in elderly men with treated leprosy. Leprosy Review 2001; 72(3): 322-329.

[13] Hardy R, Cooper MS. Bone loss in inflammatory disorders.Journal of Endocrinology 2009; 201: 309-320.

[14] Indumati V, Patil VS. Biochemical markers of bone remodeling in Osteoporosis- current concepts. Journal of clinical and Diagnostic Research 2010; 4: 2089-2097.

\section{How to cite this article:}

Yaswanth Kumar K et al Evaluation of Biochemical Markers for Bone Changes in Leprosy Patients. Int. J. Adv. Pharm. Biotech., 2018; 4(3): 1-5. 